\title{
Mass Spectrometric Studies of Thermal-Nonoxidative Degradation of Poly(vinyl chloride)
}

\author{
Zahoor AHMAD and Fazal MAHMOOD \\ Department of Chemistry, Quaid-i-Azam University, Islamabad, Pakistan
}

(Received March 21, 1996)

\begin{abstract}
Mass spectrometric studies of thermal-nonoxidative degradation of poly(vinyl chloride) (PVC) were carried out in the temperature range of $473-523 \mathrm{~K}$. The population of various series of polyene linkages at different temperatures was studied. Concentration of polyene linkages with odd numbers of carbon atoms were found to be minimal as compared to polyene linkages containing the adjacent even numbers of carbon atoms. These results are explained on the basis of strain on the valence angle and permissible ring formation and agree with those of Spanagel and Carother on depolymerization of polyesters.
\end{abstract}

KEY WORDS Poly(vinyl chloride)/ Thermal Non-oxidative Degradation / Mass Spectrometry Polyenes Yield / Spanagel Carother Theory /

Poly(vinyl chloride) (PVC) is the second most important plastic used in the world both in rigid and flexible forms. Although it has numerous excellent properties thermal degradation of PVC occurs during processing, or when it is subjected to high temperature while in use. In the early stages, dehydrochlorination of PVC takes place and long conjugated polyene linkages are formed. Rapid growth of a polyene sequence takes place probably as a consequence of allylic activation of monomeric units in the vicinity of the $\mathrm{C}=\mathrm{C}$ bond. The growth of polyene sequence ceases after reaching a certain length. This process is often called zip reaction and has been studied by various techniques including dehydrochlorination, ${ }^{1-3} \mathrm{TGA}^{4,5}{ }^{4 R},{ }^{6} \mathrm{NMR},{ }^{7} \mathrm{ESR},{ }^{8} \mathrm{UV},{ }^{9-11}$ and mass spectrometry. ${ }^{12}$ In the later stages, long polyene linkages further decompose into smaller ones and these small polyene linkages may cyclize to form cyclic compounds as shown by many workers. ${ }^{13,14}$

This paper discusses the second stage of degradation in which long polyene linkages break down into smaller ones and cyclize via intra or intermolecular interactions. The relatively large amount of polyene linkage containing even numbers of carbon atoms rather than odd numbers has been explained on the basis of a theory due to Spanagel and Carother. ${ }^{15}$

\section{EXPERIMENTAL}

Analytical grade PVC powder (BDH, England) $(M W=$ $100000 \pm 20),(100 \%$ of the powder passes through 60 mesh and $74 \%$ through 200 mesh) was used as such. PVC was dissolved in peroxide-free spectrograde tetrahydrofuran.

For mass spectrometric measurements, a sample was degraded in the pyrolysis unit of GC/MS for $15 \mathrm{~min}$ in a $\mathrm{N}_{2}$ atmosphere at the desired temperature. The volatile products of degradation were introduced to the mass spectrometer (Shimadzu GCMS QP 1000) by the vacuum system of the spectrometer. The species formed were passed through the gas chromatograph column OV-17 and were detected by the molybedinum hyperbolic quadrupole of mass spectrometer. The software of GC/MS displayed the spectra of the sample. Yields of different polyenes were differentiated by pattern recognition analysis.

\section{RESULTS AND DISCUSSION}

The results of mass spectrometeric studies shows that dehydrochlorination of PVC occurs with the formation of polyene linkages and is followed by the breaking of long polyene linkages into smaller ones. Mass spectra show a number of peaks, the strongest at $m / z$ values of $36(\mathrm{HCl}), 56$ (butene), 78 (benzene), and 92 (toluene). Peaks above $m / z=250$ have relative intensities of less than $1 \%$ of $\mathrm{HCl}$ peak. Peak for acetylene were not observed at $453 \mathrm{~K}$, however, at $483 \mathrm{~K}$ and above this temperature a small peak was observed.

Figure 1 shows the observed yield in the quadrupole MS, of polyene linkages relative to $\mathrm{HCl} v s$. temperature

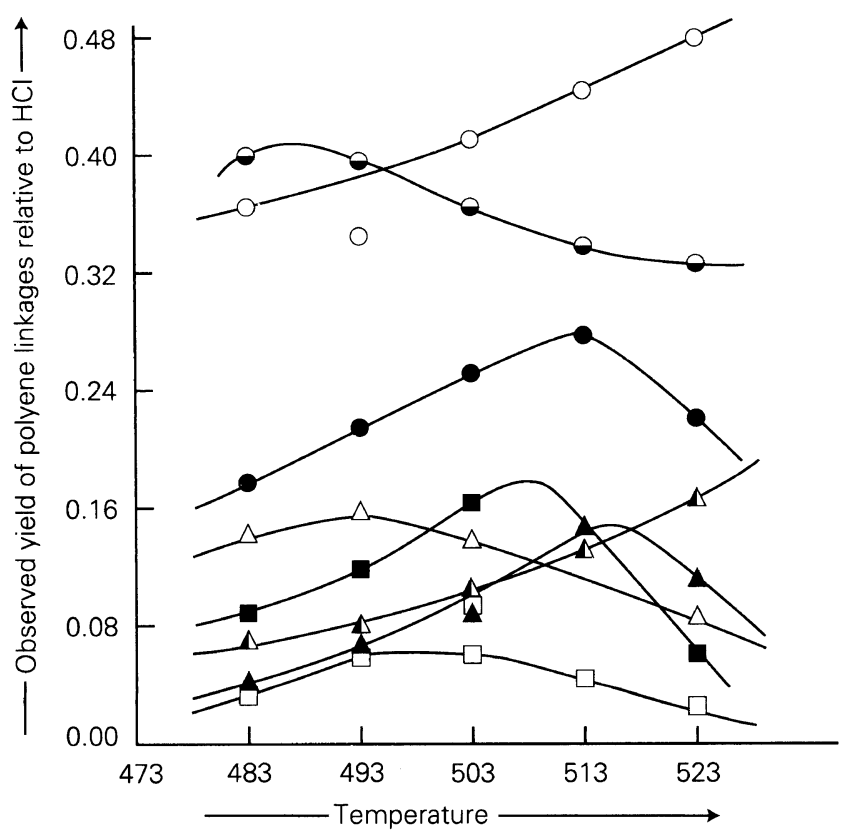

Figure 1. Variation of observed yield of polyene linkages with temperature. $\triangle, C_{3}$-polyene; $O, C_{4}$-polyene; $\Theta, C_{5}$-polyene; $O, C_{6}$ polyene; $\triangle, \mathrm{C}_{7}$-polyene; $\Delta, \mathrm{C}_{8}$-polyene; $\square, \mathrm{C}_{9}$-polyene; $\square, \mathrm{C}_{10^{-}}$ polyene. 
between 483 and $523 \mathrm{~K}$. It is evident that the concentrations of $\mathrm{C}_{3}$, and $\mathrm{C}_{4}$ polyenes increases while the concentrations of $\mathrm{C}_{6}, \mathrm{C}_{7}, \mathrm{C}_{8}$, and higher polyenes increase at first and then decrease sharply when the temperature is increased from 503 to $523 \mathrm{~K}$.

The creation of double bond as a result of degradation is rapid initially due to allylic activation of chlorine atom. Dehydrochlorination process (unzipping of the chain) give rise to polyene linkages the size of which increases first (smaller linear polyenes converting to $C_{10}$ ) but then the growth of polyene linkage stops due to resonance stabilization of the conjugated structure. It is generally believed $^{16}$ that resonance stabilization energy with $C_{10}$ and $C_{11}$ polyene reduces sufficiently the activation of allylic chlorine, but at high temperature:

i) Large polyene chains also break down producing small polyenes so the number of small polyene chains is always higher.

ii) Large polyene chains may also cyclize to produce rings. Some of these rings may be stable other not, those which are stable such polyenes may not decompose to produce smaller polyenes (see for example $\mathrm{C}_{6}$ polyenes are more than $\mathrm{C}_{5}$ or $\mathrm{C}_{7}$ ) $\mathrm{C}_{10}$ polyene are more than the $\mathrm{C}_{9}$. At low temperature cyclization process may be slow and it increases with temperature but beyond certain value, high temperature may then again decompose even such polyenes. Note the relative increase in $\mathrm{C}_{10}$ as compared to $\mathrm{C}_{9}$ with temperature.

Theoretical work applying quantum mechanical calculations by Simon et al. ${ }^{17}$ on PVC model compounds however has shown that activation energy of the dehydrochlorination process as the size of the chain grows, remains practically constant. Applying these results to PVC dehydrochlorination one may conclude that polyene linkage higher than $\mathrm{C}_{10}$ may be formed. In the present study polyene linkages higher than $C_{11}$ were not detected. It is believed that the torsional vibration of the links in polymer chain may cause deviation in the dihedral angle optimal for allylic activation thus raising the activation energy of dehydrochlorination process and limiting the size of polyene linkages up to 10 . Higher temperature also cause the motion of the links to become more intense thus breaking the chain into smaller segments, as is evident from increase in the number of small linkages in Figure 1. The small polyene linkages then may cyclize forming cyclic compounds. The cyclization of polyene linkages can occur by intramolecular interactions, giving products like benzene and toluene, or by intermolecular interactions giving products like naphthalene and methyl naphthalene. ${ }^{18,19}$ For the temperature range studied, detection of benzene and toluene shows that product formation by intramolecular interaction is more favourable than by intermolecular interactions. As the reacting groups are far apart and may not possess sufficient energy to come close and interact, so the chances of intermolecular interaction seams quite low for the temperature range studied.

Cyclization of polyene linkages by intra or intermolecular interactions depends upon the ease of formation of cyclic compounds. Spanagel and Carother ${ }^{15}$ studied the depolymerization of polyester and obseved that in the intermediate range, odd membered rings are relatively more difficult to produce than those with the adjacent

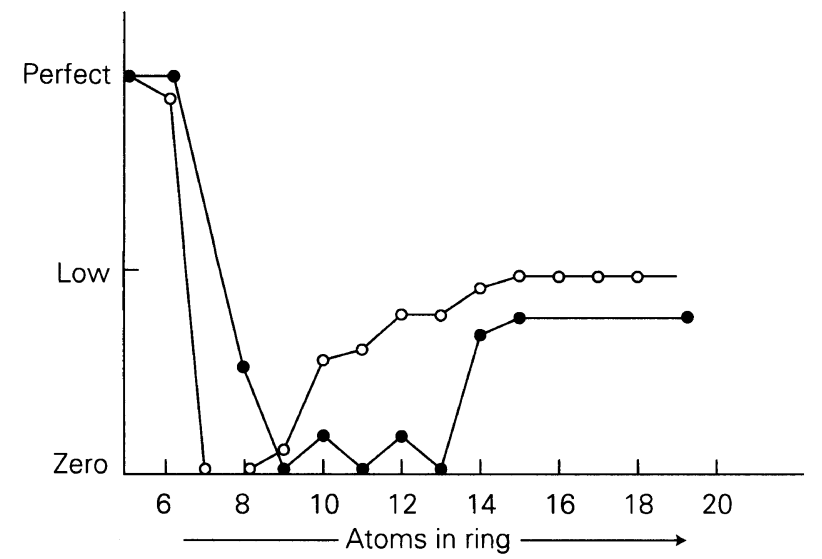

Figure 2. Ease of formation and stability vis. ring size. $-\mathrm{O}_{-} \mathrm{-}-$, ease of formation of monomeric cyclic ester of dibasic acid; - - - stability of anhydride of dibasic acid.

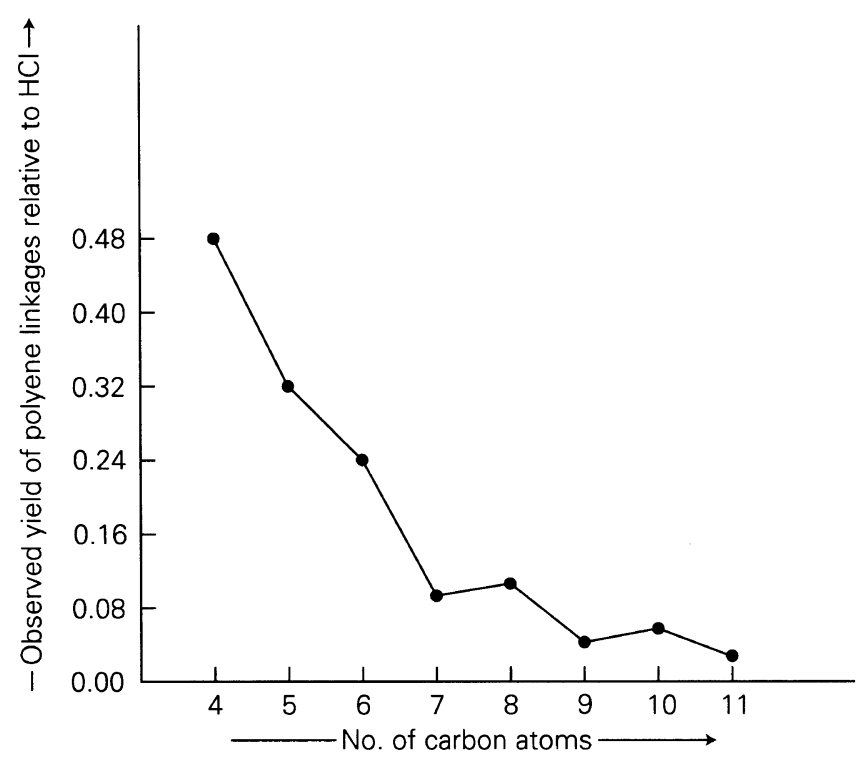

Figure 3. Yield of polyene linkages relative to $\mathrm{HCl} v s$. number of carbon atoms at $523 \mathrm{~K}$.

even numbers. They also observed that the ease of ring formation and stability in general run parallel as shown in Figure 2. According to Spanagel and Carother, strain on the valence angle and permissible ring formation are prime factors governing the size of ring. The possibility of five and six membered rings is maximum because the products are virtually strainless. The possibility of $\mathrm{C}_{9}$ and $\mathrm{C}_{11}$ cyclic compounds is minimum because strain in these compounds is maximum.

The present GC/MS studies on PVC thermal degradation have revealed that, with rise in temperature, the long polyene linkages breakdown into smaller polyene linkages. Various smaller fragments are stabilized to some extent by cyclization according to a principle explained by Spanagel and Carother.

Figure 3 shows the plot of concentration of polyene linkages $v s$. number of carbon atoms at $523 \mathrm{~K}$. The curve resembles to that obtained by Spanagel and Carother (Figure 2). Almost similar patterns were obtained at lower temperatures. The concentration of polyene linkages containing odd numbers of carbon atom were found to be minimal as compared to polyene linkages con- 
taining the adjacent even number of carbon atoms, except for $\mathrm{C}_{5}$ linkages. It seems that polyene linkages are stabilized by cyclization and the formation of cyclic compounds containing odd number of carbon atoms is more likely than that of cyclic compounds containing even numbers of carbon atoms. The Five-membered cyclic compound is virtually strainless, so its formation is favored compared to other polyene linkages containing odd numbers of carbon atoms.

The relative concentrations of the polyene linkages and the possibility of intramolecular cyclization compared to intermolecular cyclization at high temperature, will be discussed in a later paper.

\section{REFERENCES}

1. T. V. Hoang and B. Michel, Polym. Degrad. Stab., 16, 35 (1986).

2. J. Wypych, Polym. Prepr., 26, 122 (1985).

3. P. Simon and L. Valko, Chem. Prepr., 40, 509 (1984).

4. B. Satilmis and G. Olgvin, Thermo Chim. Acta, 106, 169 (1986).
5. Z. Ahmad and W. Manzoor, J. Therm. Anal., 38, 2349 (1992).

6. J. Verdu, J. Macromol. Sci. Chem., 12, 551 (1978).

7. K. B. Abbas, Pure Appl. Chem., 49, 569 (1977).

8. F. Mori, M. Kelen, B. Turcsanyl, I. Bela, and F. Tüdös, Polym. Bull., 2, 77 (1980).

9. T. T. Nagy, T. Tibor, and E. Scamporrino, J. Polym. Sci., Polym. Chem. Ed., 18, 1147 (1980).

10. W. Manzoor, S. M. Yousaf, and Z. Ahmad, Polym. Degrad. Stab., $\mathbf{5 2}$, in press.

11. W. Manzoor and Z. Ahmad, Polym. Degrad. Stab., accepted.

12. A. Ballistreri, S. Foti, G. Montaudo, . Koyama, and Y. Oki, Angew Makromol. Chem., 68, 137 (1978).

13. T. T. Nagy, T. Tibor, T. Kelen, B. Turcsanyl, I. Bela, and F. Tüdös, Polym. Bull., 3, 613 (1980).

14. J. P. Metayer, B. Becard, and J. M. Broces, Rev. Gen. Cautch. Plast., 84, 675 (1988).

15. E. W. Spanagel and W. H. Carother, J. Am. Chem. Soc., 57, 929 (1935).

16. D. Braun, Pure Appl. Chem., 26, 173 (1971).

17. P. Simon, P. Cerney, and L. Valko, Eur. Polym. J., 25, 531 (1989).

18. R. P. Lattimer, P. Robert, and J. W. Kronk, J. Appl. Polym. Sci., 25, 101 (1980).

19. J. Sliwiok and H. S. Rzepa, Microchim. J., 25, 1 (1980). 